\title{
Breakthrough against tuberculosis: high efficacy of Artemisia afra infusions
}

\begin{abstract}
Objective: The objective is to study in vivo the effect of the administration of Artemisia infusions as supplement in combination with the prescribed WHO treatment. Promising in vitro results needed to be confirmed in vivo before proceeding to larger scale clinical trials.

Methods: A first pilot study was run with 5 patients using Artemisia afra infusions and a second one on 10 patients with Artemisia annua infusions. Over 15 days their fever, diurnal and nocturnal coughing, asthenia, Ziehl-Neelsen assay were monitored.

Results: Artemisia afra infusions give faster symptoms resolution and healing. After 5 days all TB symptoms had disappeared whilst for Artemisia annua it took 10 days. The ZiehlNeelsen assay was negative after 10 days for the Artemisia afra treatment and after 15 days for Artemisia annua.

Conclusion: These observations on the administration of Artemisia infusions in combination with the conventional WHO treatment gave an astonishingly faster relief of the symptoms than conventional treatment alone. This could be a base to provide a potential natural and inexpensive therapy for TB treatment failure and resistance. More in vivo studies with a larger cohort are required to confirm our findings.
\end{abstract}

Keywords: tuberculosis, mycobacterium, Artemisia afra, in vivo
Volume 9 Issue 2 - 202 I

\author{
Bati Daddy,' Pierre Lutgen, ${ }^{2}$ Pascal Gisenya ${ }^{3}$ \\ 'Ruanguba Hospital, North Kivu Province, DR Congo \\ 2IFBV-BELHERB, Luxembourg \\ ${ }^{3} \mathrm{HEAL}$ Africa, DR Congo
}

Correspondence: Pascal Gisenya, Healafrica I I I, Lyn Lusi, Goma, North Kivu, Democratic Republic of Congo, Tel +I-587-974-76 I I,Email pbgisenya@gmail.com

Received: March 20, 2021 | Published: April 23, 2021

\section{Introduction}

Tuberculosis kills 1600000 people every year and each year there are 8 million new cases. Emergence of drug-resistant and multidrugresistant Mycobacterium tuberculosis strains is a major barrier to tuberculosis eradication, as it leads to longer treatment regimens and in many cases treatment failure. According to the 2020 report of WHO (Global tuberculosis report ISBN 978-92-4-001313-1) drugresistant tuberculosis is a major contributor to resistance worldwide and continues to be a public health threat. Annually, about half a million people fall ill with drug-resistant TB (MDR-TB) globally (Figure 1).

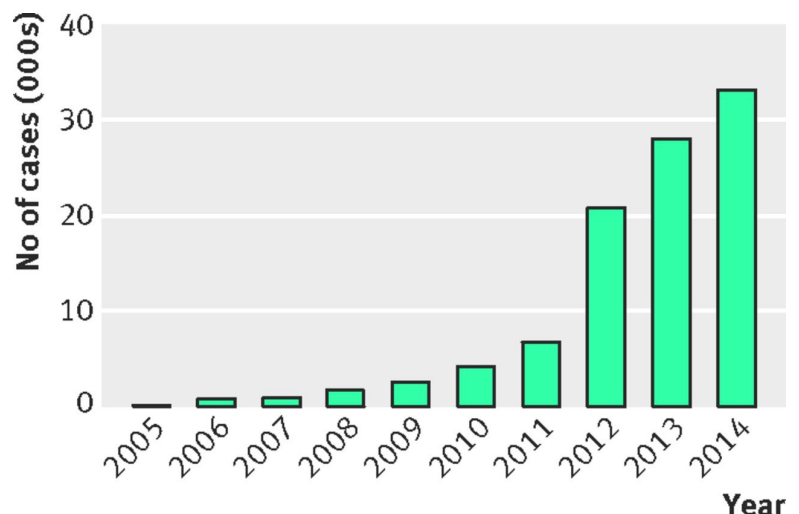

Source: WHO Global health observatory data repository

Figure I Increase of multidrug resistance against tuberculosis.

WHO published a press release on 22d March 2021. https://www. who.int/news/item/22-03-2021-covid-19-highlights-urgent-needto-reboot-global-effort-to-end-tuberculosis. The situation becomes particularly dramatic for Africa. In 2020 an additional total of 1 400000 tuberculosis infected people did not receive any treatment because of the unavailability of personnel, equipment and drugs. The death toll increased by 500000 .

"The effects of COVID-19 go far beyond the death and disease caused by the virus itself. The disruption to essential services for people with TB is just one tragic example of the ways the pandemic is disproportionately affecting some of the world's poorest people, who were already at higher risk for TB," said Dr Tedros Adhanom Ghebreyesus, WHO Director-General.

Drug resistance is a formidable obstacle to TB care and prevention globally, making it harder and longer to treat, often with poorer outcomes for patients. People with drug-resistant TB face significant economic and social costs and only 1 in 3 access quality care. Reaching the missing patients remains a significant public health challenge.

The collateral damage of resistance to existing pharmaceutical drugs will have long range dramatic consequences. As long as Mycobacterium tuberculosis patients are not cured, they will spread the disease to others. M. tuberculosis is carried in airborne particles, known as droplet nuclei that can be generated when persons with pulmonary or laryngeal tuberculosis sneeze, cough or speak. Thus, there is an urgent need to explore new TB drugs and combinations, in order to shorten TB treatment and improve outcomes. Since the failure of the MVA85A vaccine 5 years ago, there have been no new tuberculosis vaccine candidates entering clinical testing.

In clinical trials our partners have been able to document over the recent years that Artemisia plants, including Artemisia afra which does not contain artemisinin, are very efficient against several tropical diseases: Malaria, Bilharzia, Trypanosoma cruzi, Leishmaniasis. Several in vitro assays had indicated that Artemisia infusions might have a high potential in curing tuberculosis. ${ }^{1,2}$ But it happens often that in vitro results are not reproduced in vivo because of the human metabolism. The objective of our pilot trials was to confirm this potential in vivo. 


\section{Description of the pilot studies}

In September 2020, we ran a pilot trial at the Ruanguba Hospital in North Kivu Province, Democratic Republic of the Congo to confirm the efficacy of Artemisia afra as a supplement to the WHO National Treatment. We admitted 5 patients with confirmed laboratory ZiehlNeelsen test on Day 0.

All of these patients were adult male with the following symptoms typical to active tuberculosis: productive cough for more than 3 weeks aggravated at night, chest pain, fatigue, loss of appetite, fever and night sweats.

One of the patients has been on the national protocol (as per WHO recommendation: $\mathrm{R}=$ Rifampicin, $\mathrm{H}$ : Isoniazid, $\mathrm{Z}$ : Pyrazinamide, $\mathrm{E}$ : Ethambutol) for 1 month but there was no noticeable improvement in his disease.

During these trials we followed the ethical guidelines issued by WHO for TB treatments. https://www.who.int/news/item/22-03-2017who-issues-ethics-guidance-to-protect-rights-of-tb-patients. All 5 patients were admitted in isolation in a separate ward. They were given the national program treatment (RHZE) simultaneously with $10 \mathrm{gm}$ of Artemisiaafrainfusions. Theywerealso fedwith 3 mealsaday for 10 days. On day 2 of treatment the night cough was partially reduced and all the patients could sleep and continued to cough only 10 times during the day. On day 5, the following symptoms stopped: cough, fatigue, fever and night sweats. On day 7, all the patients were symptom free and displayed a lot of energy to the point that they asked to return to their fields. They walked for around $10 \mathrm{kms}$ and returned to the hospital with no symptoms. On day 10 (29/09/2020), all of the patients had sputum laboratory examination and the Ziehl-Neelsen test was negative. The results even if expected became overwhelming: total absence of mycobacteria in the sputum of the 5 patients.

In early 2021, we ran another case study, this time with Artemisia апnиa efficacy on tuberculosis, again as a supplement to the WHO conventional treatment. In February 2021, 10 people were admitted with severe tuberculosis: 4 times positive Ziehl-Neelsen test. The tables below show the outcome clinical results observations on Day 0, Day 5, Day 10, Day15 (diurnal and nocturnal coughing episodes, fever and asthenia).

Our goal was to observe the clinical outcomes after administrating the Artemisia Annua in comparison to Artemisia afra water infusions. The Artemisia annua and Artemisia afra plants are from the plantations of Dr Pascal Gisenya at Rangira, Province of North Kivu, DR Congo.
The infusions were prepared by infusing $10 \mathrm{gm}$ of the leaves and stems in 1 liter of boiled water. The daily dose was divided in 3 equal doses to be given every 8 hours.

For cough episodes and asthenia, we rated the clinical parameters as follows

40: $100 \%$

30: $75 \%$

20: $50 \%$

10: $25 \%$

$0: \quad 0 \%$

For fever: the maximum temperature was 40 degrees Celsius and the minimum 37 degrees Celsius.

On day 0: all patients of the 2 groups were Ziehl-Neelsen ++++ (4 times positive indicates a very high infection)) with uninterrupted coughing, high fever 39-40 degrees (Celsius) and a general malaise, weakness and lack of appetite.

On day 5: most patients had their fever dropping to 38 degrees, diurnal cough partially resolved, improvement in nocturnal cough and a slight return to normalcy (less asthenic).

On day 10: all of the 4 symptoms were cured with Artemisia afra and the Ziehl -Neelsen test was negative for all the patients.

On day 15: all the clinical signs were resolved for patients on Artemisia annua and the Ziehl Neelsen test was negative.

In neither of these clinical trials with Artemisia plants any side effect or toxicity was noticed. This in vivo result is to our knowledge the first observed for this treatment of tuberculosis with Artemisia afra or Artemisia annua infusions combined with conventional antituberculosis drugs.

\section{Artemisia afra treatment against tuberculosis as a supplement to WHO conventional therapy: Case studies report on 5 patients}

Tables 1-3

\section{Artemisia annua treatment against tuberculosis as a supplement to WHO conventional therapy: Case studies report on 10 patients}

Tables 4-7, Figure 2

Table I Description of clinical symptoms on day 0

\begin{tabular}{lllllll}
\hline Patient code & Gender & Age & Diurnal cough & Nocturnal cough & Fever & Asthenia \\
\hline N001 & M & 42 & 40 & 40 & 39 & 40 \\
N002 & M & 38 & 40 & 40 & 39 & 40 \\
N003 & M & 29 & 40 & 40 & 38 & 40 \\
N004 & M & 32 & 40 & 40 & 39 & 40 \\
N005 & M & 30 & 40 & 40 & 39 & 40 \\
\hline
\end{tabular}


Table 2 Description of clinical symptoms on day 5

\begin{tabular}{lllll}
\hline Patient code & Diurnal cough & Nocturnal cough & Fever & Asthenia \\
\hline N00I & 20 & 20 & 37 & 10 \\
N002 & 10 & 20 & 37 & 0 \\
N003 & 20 & 10 & 37 & 0 \\
N004 & 10 & 0 & 37 & 10 \\
N005 & 20 & 10 & 37 & 0 \\
\hline
\end{tabular}

Table 3 Description of clinical symptoms on day 10

\begin{tabular}{lllll}
\hline Patient code & Diurnal cough & Nocturnal cough & Fever & Asthenia \\
\hline N00I & 0 & 0 & 37 & 0 \\
N002 & 0 & 0 & 37 & 0 \\
N003 & 0 & 0 & 37 & 0 \\
N004 & 0 & 0 & 37 & 0 \\
N005 & 0 & 0 & 37 & 0 \\
\hline
\end{tabular}

Table 4 Description of clinical symptoms on day 0

\begin{tabular}{lllllll}
\hline Patient code & Gender & Age & Diurnal cough & Nocturnal cough & Fever & Asthenia \\
\hline N00I & M & 40 & 40 & 40 & 39 & 40 \\
N002 & F & 32 & 40 & 40 & 38 & 40 \\
N003 & M & 18 & 40 & 40 & 38 & 40 \\
N004 & M & 28 & 40 & 40 & 39 & 40 \\
N005 & M & 30 & 40 & 40 & 39 & 40 \\
N006 & F & 26 & 40 & 40 & 39 & 40 \\
N007 & M & 36 & 40 & 40 & 39 & 40 \\
N008 & M & 34 & 40 & 40 & 39 & 40 \\
N009 & M & 29 & 40 & 40 & 39 & 40 \\
N010 & F & 24 & 40 & 40 & 38 & 40 \\
\hline
\end{tabular}

Table 5 Description of clinical symptoms on day 5

\begin{tabular}{lllll}
\hline Patient code & Diurnal cough & Nocturnal cough & Fever & Asthenia \\
\hline N001 & 30 & 30 & 37 & 30 \\
N002 & 40 & 40 & 37 & 20 \\
N003 & 30 & 30 & 37 & 40 \\
N004 & 40 & 40 & 37 & 40 \\
N005 & 40 & 30 & 37 & 40 \\
N006 & 40 & 40 & 37 & 20 \\
N007 & 30 & 30 & 37 & 40 \\
N008 & 40 & 40 & 37 & 40 \\
N009 & 30 & 30 & 37 & 20 \\
N010 & 40 & 40 & 37 & 30 \\
\hline
\end{tabular}


Table 6 Description of clinical symptoms on day 10

\begin{tabular}{lllll}
\hline Patient code & Diurnal cough & Nocturnal cough & Fever & Asthenia \\
\hline N001 & 10 & 20 & 37 & 10 \\
N002 & 20 & 20 & 37 & 10 \\
N003 & 10 & 20 & 37 & 0 \\
N004 & 10 & 30 & 37 & 0 \\
N005 & 20 & 10 & 37 & 0 \\
N006 & 10 & 10 & 37 & 10 \\
N007 & 10 & 20 & 37 & 0 \\
N008 & 20 & 10 & 37 & 0 \\
N009 & 20 & 20 & 37 & 10 \\
N010 & 10 & 10 & 37 & 10 \\
\hline
\end{tabular}

Table 7 Description of clinical symptoms on day 15

\begin{tabular}{lllll}
\hline Patient code & Diurnal cough & Nocturnal cough & Fever & Asthenia \\
\hline N001 & 0 & 0 & 37 & 0 \\
N002 & 0 & 0 & 37 & 0 \\
N003 & 0 & 0 & 37 & 0 \\
N004 & 0 & 0 & 37 & 0 \\
N005 & 0 & 0 & 37 & 0 \\
N006 & 0 & 0 & 37 & 0 \\
N007 & 0 & 0 & 37 & 0 \\
N008 & 0 & 0 & 37 & 0 \\
N009 & 0 & 0 & 37 & 0 \\
N010 & 0 & 0 & 37 & 0 \\
\hline
\end{tabular}

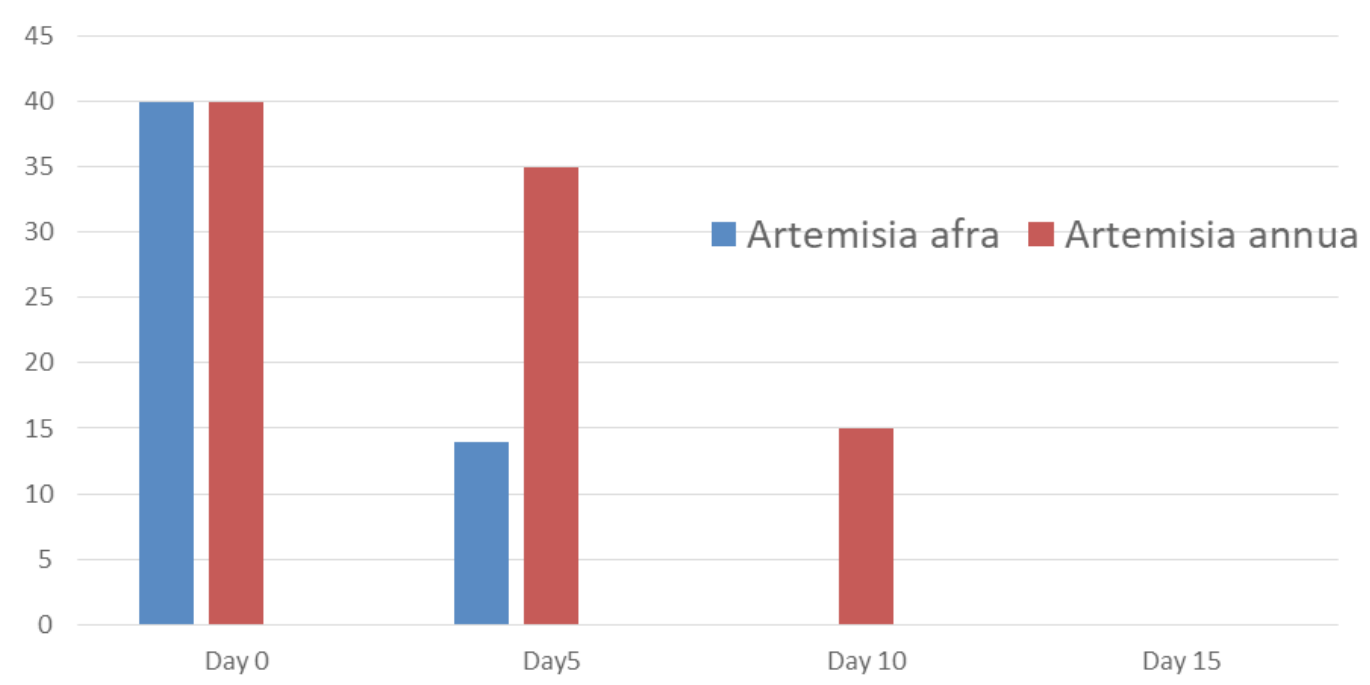

Figure 2 Diurnal and nocturnal cough (averages) for the values in the ordinate refer to the definition in the text.

Note:This figure summarizes the results for the most important symptoms listed in tables I to 7. They are also in line with those of the Ziehl-Neelsen assay (data available).

Up to now, no relapse of the disease for none of the 15 participants in the trials has been reported. 


\section{Discussion}

These observations on the administration of Artemisia infusions in combination with the conventional WHO treatment gave an astonishingly faster relief of the symptoms than conventional treatment alone. This could be a base to provide a potential natural and inexpensive therapy for TB treatment failure and resistance. In the present pilot study, the number of our patients is too small to run a statistical evaluation. More in vivo studies with a larger cohort are required to confirm our findings and the trials need to include a cohort of controls i.e. patients where only the official WHO prescribed drugs are administered.

The key questions are of course why these two Artemisia plants show this unsuspected efficacy against tuberculosis and why Artemisia afra which does not contain artemisinin has a significantly higher efficacy. We hope to elucidate this by the large-scale clinical trials we plan to run.

This study has proved that local people have a wealth of knowledge that needs to be the focal point in pharmaceutical research. Based on our own work with Artemisia and other medicinal plants over the last 10 years, we want to explore progressively several hypotheses or explanations. Artemisia afra plants contain organic compounds like luteolin, arginine, scopoletin, essential oils. We just want to quote at this stage one of our per reviewed papers on arachidonic acid. ${ }^{3}$

Too often the role of minerals has been ignored because most laboratories work with extracts obtained with organic solvents. Artemisia plants are well known to bioaccumulate minerals present in the soil. We want to investigate, based on our own work and literature data, the role of iron, zinc, selenium, potassium, gallium, silicium. ${ }^{4}$ Pharmaceutical drugs often are based on single molecules. We have data which show that in many diseases polytherapy with Artemisia plants often is more efficient than monotherapy with pharmaceutical drugs.

\section{Conclusions}

a. We believe that these breakthrough observations could be a base to provide a potential natural and inexpensive therapy for TB treatment failure and resistance. The herbal treatment is much cheaper and shorter than a regimen containing 6 months of rifampicin: $2 \mathrm{HRZE} / 4 \mathrm{HR}$ as prescribed by WHO. And no side effects or toxicities were noticed. This confirms the same innocuity previously found in other clinical trials using Artemisia infusions against malaria or schistosomiasis.

b. Artemisia afra $* *$ infusions give faster symptoms resolution and healing

c. Up to now, no relapse of the disease for none of the 15 participants in the trials has been reported. d. The findings of our pilot studies need to be confirmed by other trials, at larger scale. Currently clinical trials with Artemisia afra are planned or in progress with partners in Cameroon, Colombia, Ethiopia, Rwanda, Tanzania. There is also a need to better understand which molecules or minerals in Artemisia plants play a key role. It is possible that the positive result of our trials is due to synergy between Artemisia infusions and the conventional WHO drugs. Trials are planned where Artemisia afra infusions are administered alone without accompanying pharmaceutical drugs.

e. The authors declare that there is no conflict of interest in this study.

f. The authors thank Iwerliewen Luxembourg for the help in conducting these case studies.

** For Africa it is of high importance that Artemisia afra is a plant growing wild in many African countries, DRCongo included. Its use is perfectly legal as per WHO prescriptions (Traditional Medicine Strategy 2014-2023 and WHO/EDM/TRM/2000.1). The use of a plant where documentary evidence exists that it has been used over three or more generations for health related or medicinal purpose is perfectly legal and there is no requirement for preclinical toxicity testing. The document WHO-CDS-GMP-2019.14 recognizes that the species Artemisia afra grows through the southern and eastern parts of Africa and has been used in traditional medicine to treat a variety of ailments from asthma and rheumatism to malaria.

\section{Acknowledgments}

None.

\section{Conflicts of interest}

Authors declare that there is no conflict of interest.

\section{References}

1. Ntutela S, Smith P, Matika L, et al. Efficacy of Artemisia afra phytotherapy in experimental tuberculosis. Tuberculosis (Edinb). 2009;89 Suppl 1(Suppl 1):S33-S40.

2. Maria C Martini, Tianbi Zhang, John T Williams, et al. Artemisia annua and Artemisia afra extracts exhibit strong bactericidal activity against Mycobacterium tuberculosis. J Ethnopharmacol. 2020;262:113191.

3. Munyangi J, Pierre Lutgen. Artemisia plants, arachidonic and other polyunsaturated fatty acids. MWJ. 2020;11(3):1-8.

4. Lutgen P. Silica particles inhibit sporozoite invasion, promote $\operatorname{IgE}$, inhibit CYP3A4 and provoke bursting of infected erythrocytes. Pharm Pharmacol Int J. 2018;6(2):122-125. 